\title{
Estudio descriptivo de las desintoxicaciones de alcohol en un Servicio de Medicina Interna
}

\author{
Víctor González Vallés*; Benjamín Climent díaz**; David García Escrivá***; José Pérez- Silvestre****; \\ Pere Herrera de Pablo*****; Ma José GuiJarRo******; Agustín Herrera Ballester******* \\ *Servicio de Medicina Interna. Médico Interno Residente. Consorcio Hospital General Universitario de Valencia.
** Unidad Toxicología Clínica y Desintoxicación Hospitalaria. Serviciio de Medicina Interna. CHGUV. Coordinador.
*** Unidad Toxicología Clínica y Desintoxicación Hospitalaria. Servicio de Medicina Interna. CHGUV. Médico Adjunto.
**** Servicio de Medicina Interna. Médico Interno Residente. CHGUV.
**** Médico Interno Residente Medicina Familiar y Comunitaria. CHGUV.
**** Unidad Toxicología Clínica y Desintoxicación Hospitalaria. Servicio de Medicina Interna. CHGUV. Médico Adjunto. \\ ******S. Medicina Interna. CHGUV. Jefe de Servicio. \\ Enviar correspondencia a: \\ Benjamín Climent Díaz. Unidad de Toxicología Clínica y Desintoxicación Hospitalaria. Servicio de Medicina Interna. \\ Consorcio Hospital General Universitario de Valencia. Avda Tres Cruces 2. 46014 Valencia. E-mail: climent_ben@gva.es
}

\section{RESUMEN}

La dependencia al alcohol es una enfermedad crónica y su tratamiento comienza con la desintoxicación, continuándose con la rehabilitación. Se presenta un estudio descriptivo y retrospectivo de las desintoxicaciones de alcohol en 147 pacientes ingresados en nuestra unidad en el periodo 2003-2005. La media de edad es de 46.07 años, $77.6 \%$ hombres y $22.4 \%$ mujeres. Ingreso por síndrome de deprivación alcohólica en 31 pacientes (21.1\%), desintoxicación programada en 116 pacientes (78.9\%). Según la escala CIWA-Ar: abstinencia grado leve 100 pacientes (68.5\%), moderada 35 (24\%), grave $11(7.5 \%)$. La estancia media global fue de 11.5 días, sin diferencias estadísticamente significativas. Grupo Leve con proporción significativamente menor de hepatopatía, grupos Moderado y Grave proporción significativamente alta. Al comparar la media de la edad, estancia, cantidad de alcohol y parámetros de consumo crónico (ferritina, fe, VCM, UBE, GPT, GGT, bilirrubina y $\mathrm{Mg}$ ), se encuentran diferencias significativas entre las medias de los grupos Leve y Grave para la GPT y bilirrubina total. Se produjeron crisis epilépticas en 11 pacientes, 4 pertenecían al grupo programado, 7 con intensidad moderada-grave. No se ha evidenciado relación entre la intensidad del síndrome de deprivación, edad y sexo. Mayor intensidad en la escala CIWA-Ar (grados moderado y grave) entre los pacientes con síndrome de deprivación ya iniciado comparados con los ingresados programados, que predominantemente presentaban un grado leve. La CIWA-Ar es un instrumento útil para evaluar los síntomas de deprivación, el riesgo de complicaciones y planificar el tratamiento. La actitud clínica debe facilitar el acceso del paciente a los recursos sanitarios para el tratamiento de su adicción, normalizando la asistencia durante el ingreso hospitalario.

Palabras clave: desintoxicación, deprivación, alcohol, CIWA-Ar.

\section{ABSTRACT}

Alcohol dependence is a chronic disease whose treatment begins with detoxification, followed by rehabilitation. We present a descriptive and retrospective study of 147 patients admitted to our unit during the period 2003-2005. Median age was 46.07 years, with $77.6 \%$ men and $22.4 \%$ women. Admission diagnosis was alcohol withdrawal syndrome in 31 patients $(21.1 \%)$ and programmed alcohol detoxification in 116 patients (78.9\%). On the CIWA-Ar scale: mild withdrawal, 100 patients (68.5\%), moderate, 35 patients (24\%), severe, 11 patients (7.5\%). Mild group showed a statistically significantly lower proportion of hepatopathy, by comparison with the moderate and severe groups. Statistically significant differences were found between the mild and severe groups on comparing mean age, duration of stay, quantity of alcohol and parameters of chronic consumption (ferritine, fe, VCM, UBE, AST, bilirubin and Mg) for AST and bilirubin. There were 11 seizures: 4 patients from the programmed group, with mild withdrawal on the CIWA-Ar scale, and 7 patients with moderate-severe withdrawal. No relationship was found between patients' intensity of withdrawal syndrome, age or sex. There was higher intensity on the CIWA-Ar score (moderate and severe) among patients who had already begun withdrawal syndrome, compared to those admitted on a planned detoxification programme. The CIWA-Ar is a useful tool for assessing withdrawal symptoms and risks of complication and for planning treatment. Clinical practice must provide patients with access to health resources for the appropriate treatment of their addiction, with standardized assistance during their stay in hospital.

Key words: detoxification, withdrawal, alcohol, CIWA-Ar. 


\section{INTRODUCCIÓN}

$\mathbf{L}$ a dependencia del alcohol es una patología crónica muy prevalente con una alta morbilidad y mortalidad ${ }^{1}$. La dependencia y el consumo de riesgo de alcohol pueden causar o exacerbar numerosas enfermedades y además constituyen un factor determinante de incidentes de violencia familiar y de género, accidentes laborales y de tráfico ${ }^{2,3,4,5,6}$. Es una enfermedad crónica y su tratamiento comienza con la desintoxicación y se continua con la rehabilitación por medio de tratamiento psicoterápico, farmacológico y social 7, 9,18. El objetivo de la desintoxicación es evitar los síntomas y prevenir las complicaciones asociadas a la deprivación, hecho que se alcanza parcialmente si el síndrome ya se ha iniciado, 10

La abstinencia aguda del alcohol se puede presentar cuando un paciente con dependencia del alcohol, deja de ingerirlo durante horas. Puede presentarse ante la presencia de enfermedades concomitantes (infecciones agudas, descompensación de enfermedades crónicas, intervenciones quirúrgicas) o traumatismos agudos que requieran de ingreso hospitalario, desarrollándose progresivamente el síndrome de deprivación alcohólico. No todos los pacientes presentarán la misma sintomatología e intensidad de la misma, siendo un pequeño porcentaje los que desarrollarán sintomatología de abstinencia grave ${ }^{9}$.

El síndrome de deprivación del alcohol se caracteriza por hiperactividad vegetativa (temblor, sudoración, taquicardia, hipertensión arterial, náuseas, vómitos), ansiedad, irritabilidad, insomnio y deseo imperioso o "necesidad" de ingerir alcohol ("craving"). Cuando la deprivación es grave se pueden presentar crisis convulsivas generalizadas, agitación psicomotriz y alteraciones perceptivas (delirium tremens), que si no es adecuadamente tratada puede producir la muerte, sobretodo si el paciente presenta otros trastornos orgánicos concomitantes como traumatismo cráneoencefálico, hemorragia cerebral, neumonía, alteraciones hidro-electrolíticas, etc. ${ }^{7,19}$.

El tratamiento de desintoxicación de alcohol debe ser individualizado, adaptando los fármacos y las dosis a cada paciente según las patologías concomitantes, antecedentes y gravedad clínica7, 10, 11, 12 .

En general, se considera que la desintoxicación del paciente alcohólico se debe realizar en régimen hospitalario cuando se presentan algunos de los criterios expuestos en la tabla 17, 10,11.

El objetivo del presente estudio es describir las desintoxicaciones de alcohol de una serie de pacientes ingresados en nuestra unidad, tanto de forma programada como por inicio de síndrome de deprivación.
Tabla 1. Indicaciones de desintoxicación hospitalaria de alcohol

\begin{tabular}{|l|}
\hline $\begin{array}{l}\text { Coexistencia de patología médico-quirúrgica aguda o crónica que } \\
\text { requiera de tratamiento hospitalario. }\end{array}$ \\
\hline Riesgo de autolisis. \\
\hline Embarazo. \\
\hline Presencia de síntomas graves de deprivación a alcohol. \\
\hline Politoxicomanía. \\
\hline $\begin{array}{l}\text { Antecedentes previos de síndromes graves de deprivación a } \\
\text { alcohol con o sin delirium tremens. }\end{array}$ \\
\hline Imposibilidad de un correcto control ambulatorio. \\
\hline Antecedentes de tratamientos previos de desintoxicación \\
hospitalaria.
\end{tabular}

\section{MATERIAL Y METODOS}

Estudio descriptivo y retrospectivo de 147 pacientes ingresados en nuestra Unidad de Toxicología Clínica y Desintoxicación Hospitalaria (Servicio de Medicina Interna de un hospital terciario, integrada en la red pública de drogodependencias de la Comunidad Valenciana) en el periodo 2003-2005 para realización de una desintoxicación a alcohol, bien de forma programada o bien por inicio de síndrome de deprivación, sin excluir ningún caso.

Los pacientes atendidos proceden de las diferentes Unidades de Conductas Adictivas (UCAs), desde donde son remitidos para realización de desintoxicaciones programadas de alcohol cuando se considera que reúnen los criterios expuestos en la tabla 1. También del servicio de urgencias y otros servicios hospitalarios, cuando se ha iniciado el síndrome de deprivación a alcohol o presentan otras patologías concomitantes que hacen necesaria la desintoxicación alcohólica hospitalaria.

La cantidad de ingesta diaria de alcohol se ha medido en unidades de bebida estándar (UBE) equivalente a 10 gramos de alcohol. La intensidad del sín- 
drome de deprivación se ha valorado por medio de la escala CIWA-Ar (Revised Clinical Institute Withdrawal Assessment for Alcohol) ${ }^{13}$, considerándose la presencia de un síndrome de deprivación a alcohol leve con puntuación menor de 10, moderado con puntuación entre $10-20$ y grave por encima de 20.

Al ingreso se realiza en todos los pacientes una valoración clínica completa con anamnesis, exploración física, primera evaluación escala CIWA-Ar, determinación de tóxicos en orina, electrocardiografía y radiología de tórax.

Exceptuando los casos de ingreso urgente por inicio de síndrome de deprivación, al ingreso se informa al paciente de las características del tratamiento. La valoración por parte del servicio de Psiquiatría en los pacientes alcohólicos ingresados para desintoxicación se realiza cuando existen antecedentes de patología psiquiátrica o cuando se detecta sintomatología psiquiátrica durante el tratamiento de desintoxicación. En los casos que presentan distocia social se contacta con el Servicio de Trabajo Social para iniciar la intervención adecuada a lo largo del ingreso hospitalario.

El protocolo farmacológico habitualmente utilizado en nuestra unidad se individualiza, tanto en fármacos como en dosis, en cada caso, si bien incluían en todos los casos: diacepan, oxcarbazepina, magnesio, tiamina, ácido fólico, piridoxina, hidroxicobalamina e inhibidor secreción gástrica. La utilización de otros fármacos depende de la comorbilidad, tolerancia, alergias, etc. La administración de los fármacos, desde el inicio del ingreso hospitalario, ha sido por vía oral excepto en los pacientes en los que el control sintomático no era adecuado y presentaban un grado grave en la escala CIWA-Ar o en los casos de presentar patologías concomitantes que dificultaban el manejo de la vía oral.

Durante el ingreso hospitalario se realizan determinaciones de tóxicos y alcoholurias de forma aleatoria en tiempo y frecuencia para control de la abstinencia. Al alta hospitalaria los pacientes son controlados a los 7 días en consultas externas, continuando los controles hospitalarios hasta el alta definitiva, que dependerá de la patología orgánica que presente cada paciente.

A su vez, el paciente es remitido a su UCA para iniciar tratamiento de deshabituación, manteniendo una coordinación en el tratamiento del paciente.

Para el manejo de los datos se utilizó la herramienta informática de creación de base de datos MS Access. Las características generales de la muestra se analizaron mediante estadística descriptiva, y fueron procesadas con el paquete estadístico SPSS $\vee 12$.

El análisis descriptivo de las variables del estudio se ha realizado en función de la naturaleza de las mismas. Así, para las variables categóricas se han calculado las frecuencias (absolutas y relativas), mientras que para las cuantitativas se han calculado las medidas de tendencia central, de localización y de dispersión más habituales: media, mediana, mínimo, máximo, percentiles 25 y 75 y desviación típica (DT).

Para contrastar la independencia entre variables categóricas se ha utilizado el estadístico chi-cuadrado o el estadístico exacto de Fisher. En los casos en los que ha resultado significativo se ha cuantificado la asociación entre las variables mediante el coeficiente de contingencia y se han utilizado los residuos tipificados corregidos para interpretar el significado de la asociación detectada. Para comparar la media de variables cuantitativas en diferentes grupos se ha utilizado el procedimiento ANOVA cuando la variable cumplía las hipótesis de normalidad y homocedasticidad y, en caso de significación estadística, se ha realizado un análisis a posteriori, en concreto, el de la diferencia honestamente significativa de Tuckey. La hipótesis de normalidad se ha contrastado mediante la prueba de Kolmogorov-Smirnov y la de homocedasticidad mediante la prueba de Levene. En los casos en los que no se cumplía alguna de las hipótesis se han transformado los datos originales y se ha aplicado otra vez el procedimiento anterior. Cuando no ha sido posible encontrar una transformación adecuada se ha optado por realizar una prueba no paramétrica, en concreto la prueba $\mathrm{H}$ de Kruskal-Wallis. En todas las pruebas realizadas se ha considerado un nivel de significación $\alpha=0.05$.

\section{RESULTADOS}

La edad media era de 46.07 años (rango 22-73, D.T. $=9.42), 114(77.6 \%)$ eran hombres y $33(22.4 \%)$ mujeres. El motivo de ingreso fue el inicio de un síndrome de deprivación alcohólica en 31 pacientes $(21.1 \%)$ y la realización de una desintoxicación programada de alcohol en 116 pacientes (78.9\%). En el grupo de pacientes que ingresaron de forma programada, los criterios de ingreso fueron el fracaso del tratamiento de desintoxicación ambulatoria, la presencia de patología orgánica y psiquiátrica concomitante, la distocia social y los antecedentes de síndromes de deprivación graves. En 80 pacientes (54.4\%) se habían realizado desintoxicaciones de alcohol previas. Entre los antecedentes toxicológicos destacaban: consumo exclusivo de alcohol en 43 casos $(29.3 \%)$, alcohol y tabaco en 74 casos (50.3\%), alcohol y cocaína en 8 casos (5.4\%), alcohol, tabaco y cocaína en 22 casos $(15 \%)$

La puntuación máxima media en la escala CIWAAr para la muestra de 146 pacientes, puesto que en un caso no se recogió al ser alta voluntaria en las primeras horas, fue de 8.1 (rango 0-29, D.T. $=6.32$ ). Cuando se consideran los tres grados de intensidad 
del síndrome de abstinencia según la puntuación de la escala CIWA-Ar tenemos: grado leve 100 pacientes (68.5\%), moderado 35 pacientes (24\%), grave 11 pacientes (7.5\%). Entre los 116 pacientes que ingresaron de forma programada para desintoxicación de alcohol, 87 casos (75.7\%) presentaron un CIWA-Ar leve, 23 casos (20\%) un grado moderado y 5 casos $(4.3 \%)$ grave. Entre los 31 pacientes que ingresaron por inicio de síndrome de deprivación a alcohol, 13 casos (41.9\%) presentaron un CIWA-Ar leve, 12 casos (38.7\%) un grado moderado y 6 casos $(19.4 \%)$ grave.
En la Tabla 2 se muestra la descriptiva básica de los parámetros analíticos recogidos en el estudio.

Presentaron serología positiva para el virus de la hepatitis C 9 pacientes (6.1\%), seroconversión virus de la hepatitis $B$ en 11 pacientes (7.5\%), virus de la inmunodeficiencia humana en un paciente $(0.7 \%)$ y serología luética en 4 casos (2.7\%). Las serologías fueron negativas en el resto de pacientes.

En las radiografías de tórax realizadas se detectaron alteraciones patológicas en 16 pacientes (10.9\%) entre las que se encontraban: neumonía, derrame pleural y cardiomegalia.

Tabla 2. Descriptiva básica de los parámetros analíticos

\begin{tabular}{|c|c|c|c|c|c|c|c|c|}
\hline & $\mathrm{N}$ & Mínimo & $P_{25}$ & Media & Mediana & P75 & Máximo & D.T. \\
\hline UBE & 146 & 4.00 & 12.75 & 20.15 & 20.00 & 21.75 & 71.00 & 9.72 \\
\hline GGTP & 141 & 12.00 & 40.00 & 415.86 & 164.00 & 532.50 & 4403.00 & 648.00 \\
\hline GPT & 146 & 7.00 & 23.75 & 67.38 & 43.50 & 85.50 & 434.00 & 69.36 \\
\hline Ferritina & 130 & 8.00 & 88.50 & 345.94 & 177.00 & 387.25 & 2299.00 & 439.29 \\
\hline VCM & 140 & 80.80 & 93.00 & 97.57 & 96.85 & 101.00 & 132.00 & 6.90 \\
\hline Creatinina & 144 & 0.35 & 0.66 & 0.79 & 0.76 & 0.87 & 2.19 & 0.25 \\
\hline Glucosa & 142 & 58 & 80 & 105.34 & 89.5 & 104 & 575 & 55 \\
\hline Albúmina & 46 & 1.9 & 2.97 & 3.59 & 3.55 & 4.2 & 6.8 & 0.87 \\
\hline $\mathrm{Na}$ & 141 & 123 & 138 & 139.66 & 140 & 143 & 150 & 4.54 \\
\hline K & 137 & 2.33 & 3.85 & 4.19 & 4.2 & 4.6 & 5.7 & 0.57 \\
\hline $\mathrm{Ca}$ & 116 & 7.4 & 8.92 & 9.30 & 9.36 & 9.6 & 14.4 & 0.64 \\
\hline $\mathrm{Mg}$ & 131 & 1.3 & 1.9 & 2.08 & 2.1 & 2.3 & 3 & 0.32 \\
\hline Colesterol & 135 & 88 & 173 & 232.52 & 203 & 248 & 1945 & 188.98 \\
\hline Triglicéridos & 136 & 38 & 69 & 170.80 & 106.5 & 202.75 & 1872 & 227.63 \\
\hline GOT & 139 & 13 & 32 & 90.41 & 56 & 115 & 936 & 104.83 \\
\hline Bilirrub. Tot & 141 & 0.11 & 0.56 & 1.40 & 0.83 & 1.39 & 13.1 & 1.86 \\
\hline CPK & 85 & 23 & 56 & 1258.70 & 93 & 186.5 & 63707 & 7161.03 \\
\hline Vit B12 & 131 & 132 & 359 & 646.43 & 509 & 826 & 4690 & 506.17 \\
\hline Ac. Fólico & 132 & 0.7 & 2.9 & 6.19 & 4.2 & 8.9 & 33.4 & 5.08 \\
\hline Quick & 120 & 0.34 & 0.93 & 0.99 & 1 & 1.07 & 1.35 & 0.17 \\
\hline$H b$ & 142 & 5.3 & 12.6 & 13.74 & 13.85 & 15 & 21.6 & 2 \\
\hline Hto & 129 & 0.15 & 0.36 & 0.40 & 0.4 & 0.43 & 0.64 & 0.06 \\
\hline F. Alcalina & 134 & 5.45 & 61 & 92.55 & 79 & 105.5 & 331 & 48.44 \\
\hline TSH & 126 & 0.2 & 1.09 & 2.33 & 1.63 & 2.9 & 14.65 & 2.19 \\
\hline T4 libre & 125 & 0.7 & 1.06 & 1.3 & 1.19 & 1.37 & 8.4 & 0.72 \\
\hline Amilasa & 122 & 4.2 & 46.5 & 73.28 & 62.5 & 78.25 & 622 & 70.75 \\
\hline $\lg G$ & 34 & 0.28 & 692.75 & 887.99 & 918 & 1200 & 1860 & 473.02 \\
\hline
\end{tabular}


En los electrocardiogramas practicados se detectaron alteraciones patológicas en 16 casos (10.9\%), consistiendo en bloqueos AV de primer grado, hipertrofia ventricular izquierda, fibrilación auricular y signos de isquemia coronaria crónica. La ecografía abdominal se realizó en 45 pacientes $(30.6 \%)$ que presentaban alteraciones en los parámetros analíticos hepáticos detectándose hallazgos patológicos en 41 casos (91.1\%), consistiendo en esteatosis hepática, hepatomegalia, ascitis y signos de hipertensión portal.

Se realizaron 26 exploraciones radiológicas cerebrales con TAC o RNM (17.7\%) ante la sospecha de complicaciones agudas o crónicas de la enfermedad alcohólica, encontrándose hallazgos patológicos en 17 casos (65.4\%), consistiendo en signos de atrofia corticosubcortical y signos compatibles con encefalopatia de Wernicke. Se realizaron 11 electroencefalografías (7.5\%) en pacientes que habían presentado al ingreso o durante el mismo un primer episodio de crisis epiléptica, con resultados normales en todos los casos.

La estancia media global fue de 11.5 días (rango 1-44, D.T. $=6.17)$. No hubo diferencias estadísticamente significativas entre la estancia media del grupo que ingresó programado con respecto al grupo con síndrome de deprivación, aunque el $50 \%$ de los pacientes que ingresaron programados presentaron una estancia entre 7.25 y 13.75 días, mientras que en el grupo con síndrome de deprivación presentaban una estancia entre 9 y 16 días.

La mortalidad de esta serie de pacientes fue de 2 casos (1.4\%), siendo dos pacientes ingresados por síndrome de deprivación alcohólica, con hepatopatia crónica severa, el primero por sepsis por neumonía y el segundo por descompensación hidrópica y hemorragia digestiva alta. Se produjeron 4 interrupciones voluntarias (2.8\%) sin completar el tratamiento de desintoxicación. Había antecedentes de crisis epilépticas previas en 20 pacientes (13.6\%), todas ellas secundarias a tóxicos. Se produjeron 11 episodios de crisis epilépticas (7.5\%), 6 en el grupo de desintoxicación programada y 5 en el grupo con deprivación iniciada al ingreso, aunque no hay evidencia estadística en contra de la hipótesis de independencia entre las crisis epilépticas y el motivo de ingreso (estadístico exacto de Fisher $p=0.054$ ). Entre estos 11 pacientes, 4 pertenecían al grupo programado con un grado leve en la escala CIWA-Ar y los 7 restantes con grado moderado-grave; 9 de ellos tenían antecedentes de crisis epilépticas.

Los diagnósticos secundarios al alta hospitalaria en relación con la enfermedad alcohólica fueron: delirium tremens en 5 casos $(3.4 \%)$, encefalopatia de Wernicke en 6 casos (4.1\%), síndrome de Korsakoff en 3 casos (2\%), cirrosis hepática descompensada en 11 casos (7.5\%), hepatopatia crónica alcohólica en 90 casos (61.2\%), polineuropatía alcohólica en 13 casos (8.8\%), pancreatitis aguda en 2 casos (1.4\%), miocardiopatía dilatada en 2 casos (1.4\%), demencia alcohólica en 6 casos (4.1\%). Además, otros diagnósticos secundarios al alta hospitalaria fueron: trastorno depresivo crónico en relación con consumo de sustancias en 22 casos (15.2\%), infección respiratoria de vías bajas-neumonía en 10 casos $(6.9 \%)$, diabetes mellitus tipo 2 en 11 casos (7.6\%), dislipemia en 34 casos $(23.1 \%)$, rabdomiolisis aguda en 6 casos (4.1\%), insuficiencia renal aguda en 3 casos $(2 \%)$, dermatitis seborreica en 4 casos $(2.8 \%)$, ascitis en 5 casos (3.5\%), hemorragia digestiva alta en 2 casos (1.4\%), hipertensión arterial en 11 casos (7.6\%).

En la Tabla 3 se muestran los resultados obtenidos al contrastar la independencia entre grupo de CIWA-Ar máximo y motivo de ingreso, sexo, epilepsia por tóxicos y hepatopatía crónica alcohólica respectivamente. Para los contrastes significativos se muestra, además, el valor del coeficiente de contingencia (C) y, entre paréntesis, los residuos tipificados corregidos.

Tabla 3. Tablas de contingencia y pruebas de independencia

\begin{tabular}{|c|c|c|c|c|}
\hline & \multicolumn{3}{|c|}{ CIWA-AR máximo } & \\
\hline & Leve & Moderado & Grave & \\
\hline \multicolumn{5}{|l|}{ MOTIVO INGRESO } \\
\hline Desintoxicación & $87(3.6)$ & $23(-2.2)$ & $5(-2.8)$ & $\chi^{2}=14.92, \mathrm{gl}=2$ \\
\hline Deprivación & $13(-3.6)$ & $12(2.2)$ & $6(2.8)$ & $p=0.001, C=0.304$ \\
\hline \multicolumn{5}{|l|}{ SEXO } \\
\hline Hombre & 72 & 31 & 10 & $\chi^{2}=5.31, g l=2$ \\
\hline Mujer & 28 & 4 & 1 & $P=0.07$, n. s. \\
\hline \multicolumn{5}{|l|}{ EPILEPSIATÓXICOS } \\
\hline Sí & $4(-2.4)$ & $2(-0.5)$ & $5(5)$ & E.Fisher \\
\hline No & $96(2.4)$ & $33(0.5)$ & $6(-5)$ & $p<0.001, C=0.380$ \\
\hline \multicolumn{5}{|l|}{ HEPAT. CRÓNICA ALC. } \\
\hline Sí & $52(-3.5)$ & $27(2.2)$ & $11(2.7)$ & $\chi^{2}=14.33, g l=2$ \\
\hline No & $48(3.5)$ & $8(-2.2)$ & $0(-2.7)$ & $p=0.001, C=0.299$ \\
\hline
\end{tabular}

* Los valores entre paréntesis corresponden a los residuos tipificados corregidos

** $p=p$-valor, $C=$ coeficiente de contingencia 
El motivo de ingreso está relacionado con el grupo de CIWA-Ar máximo y además los residuos tipificados corregidos revelan que en el grupo Leve existe una proporción significativamente más alta de ingresos por Desintoxicación que por Deprivación 13,6 frente a -3,6), mientras que en los grupos Moderado y Grave existe una proporción significativamente más alta de ingresos por Deprivación que por Desintoxicación $(2,2$ y 2,8 frente a $-2,2$ y -2,8). La relación grupo CIWA-Ar máximo y convulsiones por tóxicos también es significativa, obteniendo una proporción significativamente baja de casos con convulsiones en el grupo Leve y significativamente alta en el grupo Grave. Del mismo modo también se ha encontrado relación significativa entre el grupo CIWA-Ar y hepatopatía cróni- ca alcohólica. En el grupo Leve existe una proporción significativamente menor de casos con este tipo de hepatopatía, mientras que en los grupos Moderado y Grave esta proporción es significativamente alta.

Los resultados obtenidos al comparar la media de la edad, estancia, cantidad de alcohol consumido y los distintos parámetros de consumo crónico de alcohol en los grupos de CIWA-Ar máximo se muestran en la Tabla 4.

En el análisis a posteriori de todos los contrastes significativos se encontraron únicamente diferencias significativas entre las medias de los grupos Leve y Grave en la GPT y la bilirrubina total.

Tabla 4. Medias e intervalos de confianza al $95 \%$ (IC95\%) para la media en los 3 grupos de CIWA-Ar máximo y resultado del test de comparación de medias.

\begin{tabular}{|c|c|c|c|c|c|c|c|}
\hline & \multicolumn{6}{|c|}{ CIWA-Ar máximo } & \\
\hline & \multicolumn{2}{|c|}{ Leve } & \multicolumn{2}{|c|}{ Moderado } & \multicolumn{2}{|l|}{ Grave } & \\
\hline & Media & IC95\% & Media & IC95\% & Media & IC95\% & \\
\hline Edad & 45.67 & $\begin{array}{l}L I=43.79 \\
L S=47.55\end{array}$ & 47.03 & $\begin{array}{l}L I=43.86 \\
L S=50.19\end{array}$ & 44.73 & $\begin{array}{l}L I=39.27 \\
L S=50.19\end{array}$ & $\begin{array}{l}\text { ANOVA, } \\
p=0.69\end{array}$ \\
\hline Estancia & 10.53 & $\begin{array}{l}L I=9.59 \\
L S=11.47\end{array}$ & 12.69 & $\begin{array}{l}L I=9.89 \\
L S=15.49\end{array}$ & 15.27 & $\begin{array}{l}L I=10.30 \\
L S=20.25\end{array}$ & $\begin{array}{l}\text { ANOVA, } \\
p=0.026\end{array}$ \\
\hline UBE & 19.03 & $\begin{array}{l}L I=17.49 \\
L S=20.57\end{array}$ & 22.88 & $\begin{array}{l}L I=18.45 \\
L S=27.31\end{array}$ & 23.27 & $\begin{array}{l}L I=14.52 \\
L S=32.03\end{array}$ & $\begin{array}{l}\text { ANOVA, } \\
p=0.106\end{array}$ \\
\hline $\mathrm{Mg}$ & 2.09 & $\begin{array}{l}L I=2.02 \\
L S=2.15\end{array}$ & 2.08 & $\begin{array}{l}L I=1.96 \\
L S=2.19\end{array}$ & 2.01 & $\begin{array}{l}L I=1.76 \\
L S=2.26\end{array}$ & $\begin{array}{l}\text { ANOVA, } \\
p=0.804\end{array}$ \\
\hline GPT & 57.88 & $\begin{array}{l}L I=46.36 \\
L S=69.39\end{array}$ & 82.28 & $\begin{array}{l}L I=49.42 \\
L S=115.15\end{array}$ & 107.82 & $\begin{array}{l}L I=74.86 \\
L S=140.78\end{array}$ & $\begin{array}{l}\text { ANOVA, } \\
p=0.001\end{array}$ \\
\hline GGTP & 378.15 & $\begin{array}{l}L I=244.05 \\
L S=512.26\end{array}$ & 528.06 & $\begin{array}{l}L I=295.97 \\
L S=760.15\end{array}$ & 385.89 & $\begin{array}{l}L I=271 \\
L S=499.78\end{array}$ & $\begin{array}{l}K-W \\
p=0.016\end{array}$ \\
\hline $\mathrm{Bb}$ total & 1.21 & $\begin{array}{l}L I=0.91 \\
L S=1.5\end{array}$ & 1.5 & $\begin{array}{l}\mathrm{LI}=0.71 \\
\mathrm{LS}=2.29\end{array}$ & 2.36 & $\begin{array}{l}\mathrm{LI}=0.46 \\
\mathrm{LS}=4.26\end{array}$ & $\begin{array}{l}\text { ANOVA, } \\
p=0.033\end{array}$ \\
\hline $\mathrm{Fe}$ & 138.91 & $\begin{array}{l}L I=123.66 \\
L S=154.16\end{array}$ & 108.5 & $\begin{array}{l}\mathrm{LI}=82.27 \\
\mathrm{LS}=134.73\end{array}$ & 132.14 & $\begin{array}{l}L I=52.33 \\
L S=211.95\end{array}$ & $\begin{array}{l}\text { ANOVA, } \\
p=0.05\end{array}$ \\
\hline Ferritina & 293.33 & $\begin{array}{l}L I=210.5 \\
L S=376.17\end{array}$ & 492.81 & $\begin{array}{l}L I=291.12 \\
L S=694.5\end{array}$ & 359.57 & $\begin{array}{l}L I=143.25 \\
L S=575.89\end{array}$ & $\begin{array}{l}\text { ANOVA, } \\
p=0.049\end{array}$ \\
\hline VCM & 97.11 & $\begin{array}{l}L I=95.79 \\
L S=98.42\end{array}$ & 98.1 & $\begin{array}{l}L I=95.15 \\
L S=101.04\end{array}$ & 99.89 & $\begin{array}{l}L I=96.45 \\
L S=103.33\end{array}$ & $\begin{array}{l}\text { ANOVA, } \\
p=0.423\end{array}$ \\
\hline
\end{tabular}

* LI y LS límites inferior y superior, respectivamente, del intervalo de confianza. 


\section{DISCUSIÓN}

El manejo del paciente alcohólico durante su ingreso en un hospital general presenta una serie de características importantes que deben ser tenidas en cuenta por los diferentes profesionales implicados en su atención ${ }^{10,11}$.

Es fundamental detectar la enfermedad alcohólica durante la asistencia en el servicio de urgencias o al ingreso hospitalario por cualquier patología médica o quirúrgica, para iniciar una intervención terapéutica apropiada que evite la aparición de síndrome de deprivación de manera imprevista, con el consiguiente aumento de morbi-mortalidad. No se debe olvidar que en algunos pacientes médicos y quirúrgicos puede estar oculta una dependencia alcohólica previa y no diagnosticada ${ }^{11}, 14$. En nuestra serie constatamos una mayor intensidad de la puntuación en la escala CIWA-Ar (grados moderado y grave) entre los pacientes ingresados con inicio de síndrome de deprivación comparados con los ingresados de forma programada para realizar una desintoxicación de alcohol, que predominantemente presentaban un grado leve en la escala CIWA-Ar, produciéndose un mayor grado de complicaciones orgánicas en el primer grupo de pacientes, incluyendo los dos exitus.

Se ha considerado habitualmente que la estancia media es más prolongada en los pacientes con síndrome de deprivación grave a alcohol ${ }^{15}$.

En nuestra serie no hemos encontrado diferencias estadísticamente significativas en la estancia media, debido probablemente a la presencia de varios pacientes con distocia social en el grupo de desintoxicación programada, que prolongó la estancia hospitalaria. Nuestra estancia media puede parecer algo prolongada teniendo en cuenta el periodo de 7 días recomendado para llevar a cabo una desintoxicación programada ${ }^{13,14}$

La concomitancia de patología orgánica, tanto aguda como crónica descompensada, en un alto porcentaje de pacientes que ingresan en nuestra unidad prolongan dicha estancia media habitualmente, sin olvidar la presencia de casos con distocia social que requieren de una intervención sociosanitaria específica. Al igual que otros autores ${ }^{16}$, no hemos evidenciado relación entre la intensidad del síndrome de deprivación alcanzado, la edad y el sexo de los pacientes. A pesar de que la cantidad media diaria de alcohol ingerido era mayor en el grupo con puntuación grave en la escala CIWA-Ar, no había significación estadística en esta diferencia, aunque no se ha considerado el tiempo de consumo de alcohol con criterios de dependencia ${ }^{17}$.

Entre los marcadores de consumo crónico de alcohol sólo la GPT y la bilirrubina total presentaron diferencias estadísticamente significativas entre los grupos con intensidad leve en la escala CIWA-Ar y grave. Cuando se relaciona el diagnóstico de hepatopatia crónica con la intensidad de la escala CIWA-Ar, vemos que existe una relación, con presencia significativamente más alta de hepatopatia crónica en los grupos moderado y grave con respecto al leve.

La presencia de hepatopatia crónica alcohólica, que puede ser agravada por la concomitancia de infección crónica por el virus de la hepatitis C, complica el manejo del paciente alcohólico tanto por la administración de fármacos como por la probable mayor gravedad del síndrome de deprivación y complicaciones orgánicas secundarias ${ }^{17}$.

Se ha relacionado la hipomagnesemia habitual en el paciente alcohólico crónico con la intensidad del síndrome de deprivación y la hiperexcitabilidad neuronal con la aparición de crisis epilépticas ${ }^{18}$. Se han comparado las magnesemias medias en los tres grupos (leve, moderado y grave) y no se han encontrado diferencias estadísticas.

La crisis epiléptica generalizada secundaria a la deprivación de alcohol es una complicación que oscila según autores entre un 5 y $10 \%$ de los pacientes con dependencia de alcohol ${ }^{19}$. Los factores de riesgo para la aparición de una crisis epiléptica en la desintoxicación de alcohol no han sido aclarados completamente, habiéndose relacionado con la cantidad de alcohol ingerido, el tiempo de consumo, alteraciones hidroelectrolíticas y gravedad de la deprivación ${ }^{19}$. Las convulsiones pueden aparecer sin necesidad de desencadenarse un síndrome grave de deprivación con delirium, tal como hemos constatado en nuestra serie. En nuestra serie la mayoría de las crisis epilépticas se produjeron en pacientes con antecedentes previos de crisis. Esto resalta la necesidad de identificar a dichos pacientes al ingreso hospitalario para administrarles una terapia antiepiléptica de mayor intensidad.

La CIWA-Ar es un instrumento útil para evaluar la presencia y gravedad de los síntomas de deprivación, evaluar el riesgo de presentar una complicación del síndrome de abstinencia y para calcular y planificar el tratamiento farmacológico necesario en función de los síntomas presentes.

Algunos autores han propugnado una actitud terapéutica basada en la intensidad del síndrome de deprivación evaluado por la CIWA-Ar sin seguir un esquema de posología farmacológica fijo 8, 10, 11, 12, 20, 21, 22 .

En nuestra experiencia, la dosificación de los fármacos es individualizada según una serie de características de los pacientes (tolerancia a las benzodiacepinas, comorbilidad orgánica y psiquiátrica, fármacos concomitantes, antecedentes toxicológicos) junto a la intensidad del síndrome de deprivación evaluado diariamente con dicha escala ${ }^{23}$, administrándose los fármacos desde el inicio del ingreso hospitalario. La 
utilización de fármacos anticonvulsivantes como la oxcarbazepina en la desintoxicación de alcohol y otras adicciones se ha ido extendiendo en los últimos años como fármaco para prevenir y/o tratar crisis epilépticas, como estabilizador del humor y efecto sedante, que permita disminuir las dosis de benzodiacepinas durante la desintoxicación y la retirada de éstas durante la deshabituación. A pesar de su amplio uso en la práctica clínica diaria en las adicciones, no se han realizado ensayos clínicos aleatorizados y doble-ciego que aclaren su potencial papel terapéutico en esta patología7, 24, 25, 26. Aunque el presente estudio no valora la eficacia del tratamiento farmacológico en la desintoxicación de alcohol, en nuestra experiencia, la utilización de la oxcarbazepina junto a benzodiacepinas ha permitido una reducción de las dosis de estas últimas y ha facilitado su supresión tras el proceso de desintoxicación.

Las patologías orgánicas durante el ingreso hospitalario se diagnostican y tratan concomitantemente al tratamiento de desintoxicación hospitalaria, manteniendo una visión integral del paciente alcohólico, tanto al ingreso como en los controles posteriores al alta.

Constatamos un porcentaje importante de sintomatología ansioso-depresiva crónica en los pacientes alcohólicos. Aunque en nuestra unidad no disponemos de una intervención diagnóstico-terapéutica psicológica durante la desintoxicación, su disponibilidad mejoraría la transición hacía el tratamiento de deshabituación ${ }^{27,28}$

La actitud clínica ante estos pacientes debe de facilitar su acceso a los recursos sanitarios para el tratamiento de su adicción y de las complicaciones orgánicas, psicológicas y sociales secundarias a la misma, tanto durante el ingreso como al alta hospitalaria, normalizando la asistencia del paciente alcohólico durante el ingreso hospitalario.

\section{AGRADECIMIENTOS}

A Olga Cuesta por su colaboración en el estudio estadístico realizado.

\section{REFERENCIAS}

1. Anderson P, Baumberg B. El alcohol en Europa. Una perspectiva de salud pública. Institute of Alcohol Studies. Reino Unido. 2006.

2. Corrao G, Bagnardi V, Zambon A, La Vecchia C. A metaanalysis of alcohol consumption and the risk of 15 diseases. Prev Med 2004; 38: 613-619.
3. Boffetta P, Hashibe M. Alcohol and cancer. Lancet Oncol 2006; 7: 149-156.

4. Willner LR, Reuben A. Alcohol and the liver. Curr Opin Gastroenterol 2005; 21: 323-330.

5. Harper C, Matsumoto I. Ethanol and brain damage. Curr Opin Pharmacol 2005; 5: 73-78.

6. http://www.who.int/whr/2002/en/Chapter5S.pdf

7. Guardia J, Jiménez-Arriero MA, Pascual F, Flórez G, Contel M. Guía Clínica para el tratamiento del alcoholismo. Socidrogalcohol. Valencia 2007.

8. Ozdemir V, Bremner KE, Naranjo CA. Treatment of alcohol withdrawal syndrome. Ann Med 1994; 26: 101-5.

9. Ceccanti M, Balducci G, Attilia ML, Romeo M. Diagnóstico, aproximación terapéutica y rehabilitación del alcohólico. Adicciones 1999; 11: 363-372.

10. Paille F, Bazot M, Favre JD. Objetivos, indicaciones y modalidades de desintoxicación en los alcohólicos. Adicciones 2000; 12: 245-254.

11. Valdés-Stauber J. Estrategias en el tratamiento de desintoxicación alcohólica. Adicciones 2003, 15: 351368

12. Mayo-Smith MF. Management of alcohol intoxication and withdrawal. In Grahan AW, Schultz TK, Wilford BB. Principles of addiction medicine. $2 d$ ed. Chevy Chase, Md: American Society of Addiction Medicine. 1998: 431-440.

13. Sulivan JT, Sykora K, Schneiderman J, Naranjo CA, Sellers EM. Assessment of alcohol withdrawal: the revised Clinical Institute Withdrawal Assessment for Alcohol Scale (CIWA-Ar). Br J Addict 1989; 84: 13531357.

14. Lohr RH. Treatment of alcohol withdrawal in hospitalizad patients. Mayo Clin Proc 1995; 70: 777-782

15. Chang PH, Steinberg MB. Alcohol withdrawal. Med Clin North Am 2001; 85: 1191-1212.

16. Wetterling T, Driessen M, Kanitz RD, Junghanns K. The severity of alcohol withdrawal is not age dependent. Alcohol and Alcoholism 2001; 36: 75-78.

17. Wright T, Myrick $H$, Henderson S, Peters $H$, Malcolm R. Risk factors for delirium tremens: a retrospective chart review. Am J Addict 2006; 15: 213-219.

18. Wilson A and Vulcano B. A double-blind, placebocontrolled trial of magnesium sulphate in the ethanol withdrawal syndrome. Alcohol Clin Exp Res 1984; 8: 542-545.

19. Schuckit M, Tipp J, Reich T, Hesselbrock V, Bucholz K. The histories of withdrawal convulsions and delirium tremens in 1648 alcohol dependent subjects. Addiction 1995; 90: 1335-1347.

20. Ait-Daoud N, Maolcolm RJ, Johson BA. Symptomtriggered vs fixed-schedule doses of benzodiacepine for alcohol withdrawal. Arch Intern Med 2002; 162: 1117-1121.

21. Mayo-Smith MF, Beecher LH, Fischer TL, Gorelick DA, Guillaume JL, Hill A et al. Working Group on the Management of Alcohol Withdrawal delirium. An evidence-based practice guideline. Arch Intern Med 2004; 164: 1415-1412. 
22. Soler PA, Guardia J. Tratamiento farmacológico: la desintoxicación. En: Monografía Alcohol. Ed. Pascual F, Torres M, Calafat A. Adicciones 2002, 13: 421-438.

23. Puz Ca, Strokes SJ. Alcohol withdrawal syndrome: assessment and treatment with the use of the Clinical Institute Withdrawal Assessment for Alcohol-revised. Crit Care Nurs Clin North Am 2005; 17: 297-304.

24. Polycarpou A, Papanikolaou P, loannidis JPA, Contopoulos-loannidis DG. Anticonvulsivantes para el síndrome de abstinencia alcohólica (Revisión Cochrane traducida). En: La Biblioteca Cochrane Plus, 2006 Número 1. Oxford: Update Software Ltd.

25. Ait-Daoud N, Maolcolm RJ, Johson BA. An overview of medications for the treatment of alcohol withdrawal and alcohol dependence with an amphasis on the use of older and newer anticonvulsivants. Addict Behav 2006; 31: 1628-1649

26. Brett Y. Lu, Coberly R. Use of oxcarbazepine in outpatient alcohol detoxification. Am J Addict 2005; 14 : 191-192.

27. Johnson M, Brems C, Mills M, Fisher D. Psychiatric symptomatology among individuals in alcohol detoxification treatment. Addictive Behaviors 2007.

28. Casas M, Guardia J. Patología psiquiátrica asociada al alcoholismo. Adicciones 2002, vol 14 supl 1.

29. Diagnostic and statistical manual of mental disorders (DSM-IV-TR). $4^{\text {th }}$ edn., text revision. American Psychiatric Association, Washington, DC, 2000, pp 212214. 
